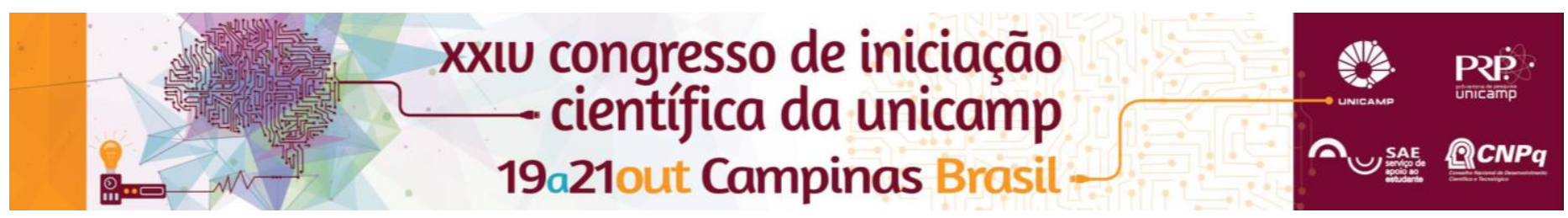

\title{
Incorporation of AgSD in KGM asymmetric membranes for wound dressing
}

\author{
Natália Osiro*, Giovana M. Genevro* e Marisa M. Beppu
}

\begin{abstract}
The development of effective and accessible treatment for 2 nd degree burns is rather relevant because of the high frequency of accidents involving this kind of injury in domestic and work environment. The objectives of this project were to produce and evaluate silver sulfadiazine (AgSD) loaded konjac glucomannan (KGM) membranes as potential wound dressings for the treatment of 2 nd degree burns. Thermal analysis revealed that sulfadiazine forms hydrogen bonds with the hydroxyl groups of KGM and that silver ions reduce to metallic silver microparticles at low temperatures. However, atomic absorption showed that a significant portion of the incorporated silver was lost in the water used to neutralize the $\mathrm{pH}$ of the membranes.
\end{abstract}

\section{Key words:}

Glucomannan, burns, silver sulfadiazine.

\section{Introduction}

Asymmetric polymeric membranes stand out as wound dressings due to its singular capacity to provide occlusion, humidity retention and antimicrobial activity simultaneously during the treatment. Those membranes consist, basically, in biocompatible polymers with two layers: A dense, for occlusion, and a porous, for exudate absorption and gaseous exchange. Antimicrobial agents, like AgSD, can be incorporated into the polymeric matrix and slowly released on the injury by both layers.

KGM is a polysaccharide extracted from the tuber of the plant Amorphophalus konjac and its composition is mainly mannose and glucose ${ }^{1}$. It has been gaining prominence in regenerative medicine due to its malleability, biocompatibility and capacity of gel formation.

\section{Results and Discussion}

KGM asymmetric membranes with 1.5 wt\%, 2 $w t \%$ and 3 wt $\%$ of AgSD and pure ones were produced by casting followed by freezing. The drug was incorporated directly into the polymeric solution. As alkali was used to aggregate the molecular chain of KGM, a neutralization process was needed.

Thermal analysis were employed to investigate the interaction between the drug and the polymer. Thermogravimetry (TGA) indicated two peaks of weight loss: At $80^{\circ} \mathrm{C}$ and around $340^{\circ} \mathrm{C}$. The first one, less intense, is due to dehydration, while the second, which is the overlap of two peaks, is attributed to the polymer's thermal degradation ${ }^{2}$. The samples with AgSD had smaller weight losses by dehydration and their thermal degradations occured at higher temperatures, what suggests that the drug stabilizes the polymer by interacting with its hydroxyl groups. Differential scanning calorimetry (DSC) of the membranes that contained AgSD shows an exothermic event without weight loss around $30^{\circ} \mathrm{C}$. Its absence in the analysis of the pure KGM membrane suggested that AgSD possibly suffers termooxidative degradation, leading to the formation of metallic silver microparticles ${ }^{3}$.

The incorporation of silver into the polymer was evaluated by measuring its concentration in the neutralization waste water using atomic absorption espectrometry method. The atomic absortion results (figure 1) demonstrated an almost complete loss of silver during the neutralization process.

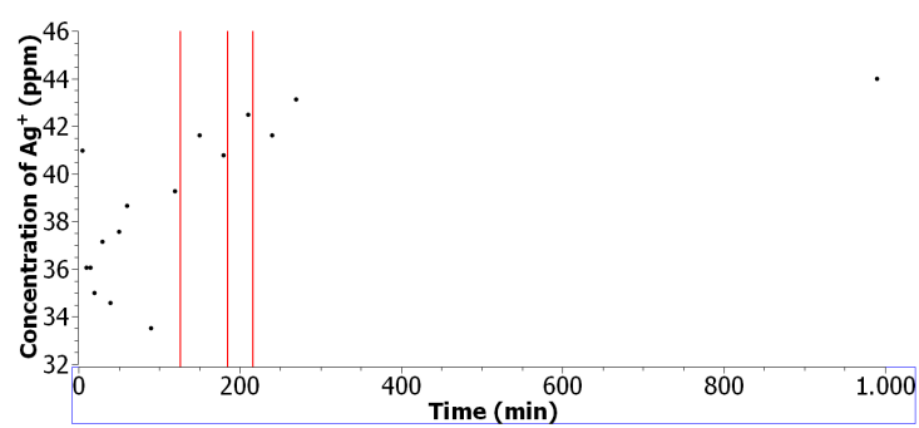

Figure 1: Concentration of $\mathrm{Ag}^{+}$in neutralization waste water from neutralization process

\section{Conclusions}

It is possible to infer, based on the results obtained, that AgSD interacts in two different ways with the polymeric matrix: The organic moiety interacts with KGM's hydroxyl groups by hydrogen bonds, while the silver, which is possibly in its metallic form, doesn't stablish an interaction strong enough to provide a drug delivery system.

Therefore, in order to use the KGM membranes as wound dressings, it is necessary to develop alternative methods to incorporate AgSD into the polymeric structure.

\section{Acknowledgement}

To CNPq and Fapesp, for the financial support.

\footnotetext{
1 Katsuraya K.; Okuyamab, K.; Hatanakab, K.; Oshimab, H.; Satoc, T. and Matsuzakic, K.. Constitution of konjac glucomannan: Chemical analysis and ${ }_{13}$ C NMR spectroschopy. Carbohydrate polymers 53 (2003) 183-189.
}

${ }_{2}$ Moriana, R.; Zhang, Y.; Mischnick, P.; Li, J; and Ek, M. Thermal degradation behavior and kinetic analysis of spruce glucomannan and its methylated derivatives. Carbohydrate polymers 106 (2014) 60-70.

3 Rosu, D.; Rosu, L and Brebu, M. Thermal stability of sulphathiazole-epoxy resin network. Journal of Analytical and Applied Pyrolysis 92 (2011) 10-18 